\title{
ZUR EINFÜHRUNG
}

Im Herbst 1934 wandte sich der am 13. Juli 1934 ins Leben gerufene, unter der Leitung des Herrn Staatsrat Geh. Regierungsrat Dr. Theodor Wieg and stehende Wissenschaftliche Ausschuß des Deutschen Orient-Vereins an die in Berlin tätigen orientalistischen Vereinigungen zum Zwecke der gemeinsamen Einladung zu einer Vortragsreihe 'Die deutsche Orientforschung, ihre Gegenwartsbedeutung und ihre Gegenwartsaufgaben'. Die Aufforderung erging an die Ortsgruppe der Deutschen Morgenländischen Gesellschaft, die Vorderasiatisch-Ägyptische Gesellschaft, die Deutsche Orient-Gesellschaft, die Altorientalische Gesellschaft, die Deutsche Gesellschaft für Islamkunde, die Gesellschaft für Ostasiatische Kunst und den Verband für den fernen Osten. Die genannten Vereinigungen gaben bereitwillig ihre Zustimmung und verzichteten zum größeren Teil zugunsten des gemeinsamen Unternehmens auf eigene Vortragsveranstaltungen während des bevorstehenden Winters.

Zu dem Unternehmen ermutigten die Worte der Anerkennung und das Versprechen kräftiger Förderung seitens des Staates, die der Reichserziehungsminister bei Gelegenheit der Berliner Jahrtausendfeier zum Gedächtnis Firdosis am 27. September 1934 durch den Mund seines Vertreters der deutschen Orientforschung mitgeteilt hatte. Man findet sie in der Zeitschrift der Deutschen Morgenländischen Gesellschaft Bd. $88 \mathrm{Heft} 2 \mathrm{~S}$. I $12-$ I I 5 wiedergegeben. Kurz vorher hatte der 7. Deutsche Orientalistentag zu Bonn (29. August bis I. September) ein eindrucksvolles Zeugnis für die Einheit und Solidarität der Orientforschung abgelegt. Anstatt der sonst üblichen Aufteilung der wissenschaftlichen Vorträge in fast beziehungslos nebeneinanderstehende Fachgruppen war eine einheitliche Reihe von Vorträgen aufgestellt worden, an 
denen sämtliche Besucher der Tagung teilnahmen. Es wurde dem Sinologen Gelegenheit geboten, sich über neue Ergebnisse der vorderasiatischen Altertumskunde zu unterrichten, während der Islamforscher Neues aus der Indologie erfuhr - und der Erfolg bewies die Richtigkeit dieser Neuordnung. So wurde der Wunsch rege, über den Kreis der Fachgenossen hinauszugehen und einem größeren gebildeten Publikum in der Reichshauptstadt Rechenschaft über Ertrag und Bedeutung der orientalistischen Arbeit abzulegen.

Die Hoffnungen, mit denen die Veranstalter das Unternehmen begleiteten, wurden durch die Aufnahme, die den Vorträgen zuteil wurde, und durch die warme und gleichbleibende Teilnahme der großen Hörerschar über Erwarten erfüllt. Die weite Verbreitung des Interesses an den Fragen des Orients in Geschichte und Gegenwart wurde dadurch ebenso ins Licht gerückt wie das Verlangen nach sachlich unvoreingenommener und objektiver Darlegung geschichtlich-gegenwärtiger Dinge überhaupt.

Den vielfach laut gewordenen Wunsch, die Vorträge im Druck festzuhalten, hat der Wissenschaftliche Ausschuß des Deutschen Orient-Vereins sich zu eigen gemacht. Er fand bei der Verlagsbuchhandlung bereitwilliges Entgegenkommen und beauftragte den Unterzeichneten mit der Herausgabe. Die Vorträge erscheinen hier, wie sie gehalten wurden; nur beim dritten Vortrag beschränkt sich die Mitteilung auf den ersten, das Grundsätzliche zusammenfassenden Teil, unter Fortlassung der die anschließenden Lichtbilder begleitenden Erläuterungen. Für die einzelnen Vorträge tragen die Verfasser die wissenschaftliche Verantwortung.

Berlin, im März 1935 .

Hans Heinrich Schaeder 